\title{
Corrosion Reliability Assessment of Underground Water Transmission Pipelines by IHS Algorithm
}

\author{
N. Kazemi ${ }^{1 *}$, S. Sanayee Moghadam² ${ }^{2}$ A. Ghasemi and H. Abotorab ${ }^{4}$ \\ ${ }^{1}$ Department of engineering and development, ABFASB. Co., Zahedan, Iran \\ ${ }^{2}$ Executive and Technical Expert, Abfan Consultant Eng. Co., Tehran, Iran \\ ${ }^{3}$ Managing Director, Water \& Wastewater Eng. Co., Tehran, Iran \\ ${ }^{4}$ Chief Research \& amp; Technical Officer, National Water \& amp; Wastewater Eng. Co, Zahedan
}

\begin{abstract}
Lifetime of pipelines is very important for safe and sanitary water transmission pipelines and water distribution networks. For this purpose, reliability assessment analysis is a good tool and has made it easy or feasible to make better decisions for inspections during maintenance and utilization process. In this study, a non-linear state model of corrosion has been used for the structural analysis of corroded water transmission pipelines, stressed by internal pressure and also substance corrosion has beenconsidered simultaneous base on a limit state function. In order to take the uncertainty associated with the design and environmental variables into account and to obtain failure probability (reliability index), an improved harmony search meta-heuristic optimization algorithm is selected. Sensitivity analysis of associated parameters is carried out to measure the effectiveness of each parameteron the probability of pipe failure. Results obtained for steel pipeline of Karevandar to Kash water transmission project arediscussed as a case study.
\end{abstract}

Keywords: Water transmission pipelines; Corrosion reliability assessment; Harmony Search Optimization Algorithm; Failure probability

Nomenclature and Units

\begin{tabular}{|ll|}
\hline PDF & Probability Density Function \\
\hline$l s f$ & Limit State Function \\
\hline$F_{X}(x)$ & Probability Distribution Function \\
\hline$Z$ & Performance of Structure \\
\hline$P_{f}$ & Probability of Failure \\
\hline FORM & First Order Reliability Method \\
\hline$S O R M$ & Second Order Reliability Method \\
\hline$M C$ & Monte Carlo Method \\
\hline$B_{H L}$ & Hasofer-Lind Reliability Index \\
\hline$C D F$ & Cumulative Distribution Function \\
\hline$H S A$ & Harmony Search Algorithm \\
\hline$I H S$ & Improved Harmony Search \\
\hline$H M S$ & Harmony Memory Size \\
\hline$H M C R$ & Harmony Memory Considering Rate \\
\hline$P A R$ & Pitch Adjustment Rate \\
\hline$C O V$ & Coefficients of Variation \\
\hline
\end{tabular}

\section{Introduction}

Generally, in the engineering problems, we face the parameters and variables which are not fixed and deterministic. They inherently have random natures that makes assessment of these problems uncertain. Due to these uncertainties, the need to scientific and systematic methods to assess reliability and predict failure

*Corresponding author. Tel.: +98 9151901821, Fax: +98 33445192 E-mail address: kazemi.na@abfasb.ir. probability of the structures is inevitable. In sensitive infrastructures such as liquid transmission pipelines (e.g. gas, oil or water...), this issue plays a more important role. As we know, water pipelines are one of the most commonly applied means of transporting water all around the world. The increasing number of aging pipelines in operation has dramatically soaredthe number of accidents. One of the major reasons of accidents in water pipelines is corrosion defect. As a pipeline ages, it can be affected by a corrosion mechanisms, which may lead to a reduction in its structural integrity and eventual failure. So, regular inspections of pipelines can decrease the risk of any undue accident. So, we are faced with a statistical problem and decision-making for reliability assessment of repairing or replacing pipelines. Studies developed by Kiefner [1] and Kiefner and Vieth [2] resulted in the well-known ASME B31G criterion [3]. Det Norske Veritas published recommended practices for assessing corroded pipelines under combined internal pressure and longitudinal compressive stress [4]. Based on both experimental tests and numerical calculations, the proposed empirical formulae comprise single and interacting defects, and complex-shaped defects. Nahal and Khalif researched the corrosion of pipeline and published their work titled "Failure Probability Assessment for Pipelines under the Corrosion Effect". They found that both defect depth and fluid pressure have significant influences on pipeline reliability [5]. In 
this study, a non-linear state model has been used for the structural analysis of corroded pipelines, stressed by external forces. External load and substance corrosion are considered simultaneous base on limit state function. In order to take the uncertainty associated with the design and environmental variables into account and obtain failure probability (reliability index), a harmony search meta-heuristic optimization algorithm has been selected. Sensitivity analysis of associated parameters is carried out to measure the effectiveness of each parameter on the probability of pipe failure. Results obtained for a steel water pipeline of Kash water transmission project are discussed as a case study.

\section{Reliability Assessment}

In this section, the problem of reliability assessment is stated. Let $x$ denote basic random variable defining the external loads, material properties, and the geometry of a structures and $n$, its dimension. This vector may be modeled by random vector $X$, and $f_{X}(x)$ is its probability density function (PDF). Also, structure failure state is denoted by a limit state function (lsf) $G$, and conventionally in the literature [6] with the following properties:

$G: R^{n} \rightarrow R$

$X \mapsto G(X)$

Such that:

$\operatorname{if} G(X)<0$ the system is in a failure state,

$\operatorname{if} G(X)=0$ the system is in a limit state,

if $G(X)>0$ the system is in a safe state.

The random variable defined by $Z=G(X)$ is also called the performance of the structure or the safety margin of the structure. According to the definition of $G$, when the structure margin is lower or equal to zero, the system fails. Therefore, the failure domain is defined by $D=\left\{x \in R^{n}, G(x) \leq 0\right\}$ (considering that the border $\delta(D)$ is also a failure set) [6]. The structure failure probability $P_{f}$ is given by the formula,

$P_{f}=\int_{D} f_{X}(x) d x$

Generally, it is difficult to solve Eq.1 because of one of the two following reasons:

The border $\delta(D)$ is non-liner, or the vector $X$ is not a linear function of standard Gaussian vector (i.e., vector $X$ components are not Gaussian or they are correlated). For solving this problem, intensive research have been done. A list of main approaches are mentioned as follows: Monte-Carlo simulations [8], discrete approximation [9], FORM/SORM using response surface method or the reliability index [10], and direct integration of PDF on the failure domain. The recent state-of-the-art approach on structural reliability analysis gives a good insight of most of these methods [11].

\subsection{Reliability index}

In this paper, we use the Hasofer-Lind (HL) reliability index for the assessment reliability of structures. HL reliability index is proposed in a $\Lambda$ space, where the vector components are Gaussian standard. Let $U$ be any vector of this space, $\Gamma$ the n-dimensional surface defined by the limit state function $G$ in the physical variables space $\Psi$, and $\sum=T(\Gamma)$ its image in the standard Gaussian space $\Lambda$.

The Hasofer-Lind reliability index $\left(\beta_{H L}\right)$, is defined as $\beta_{H L}=\operatorname{Min}-(d(O, M))$, where $\mathrm{O}$ is the center (origin) of space $\Lambda$, and $\mathrm{M}$ evolves on surface $\Sigma$. The transformation $T(X)=U$, may be obtained by $T_{i}(X)=U_{i}(i=1, \ldots, n),[6,12]$.
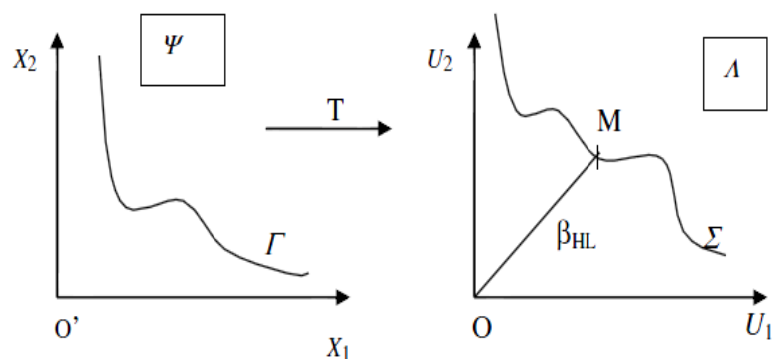

Fig. 1. Hasofer-lind reliability index

There are three main transformations $T$ which enable us to change a vector $X$ of the physical variables space $(\Psi)$ into a vector $U$ of the standard Gaussian space $(\Lambda)$ :

Rosemblatt transformation: used when the $P D F$ of $X$ is known, and when $X$ components are correlated. The components of vector $X$ are not necessary Gaussian [6].

Nataf transformation: used when PDF of $X$ is not known, but the correlation matrix of $X$ is known [11].

Plain linear transformation: used when the vector $X$ is Gaussian without any correlation between its components. Therefore, if $\mu_{i}$ and $\sigma_{i}\left(\sigma_{i} \neq 0, i=\right.$ $1,2, \ldots, n)$ are, respectively, the mean value and the standard deviation of component $X_{i}$ of vector $X$, the transformation $T(X)=U$, may be obtained by $T_{i}(X)=$ $U_{i}(i=1,2, \ldots, n)$ with $U_{i}=\left(X_{i}-\mu_{i}\right) / \sigma_{i}$. (Fig. 1).

From these, one can obtain the surface $\sum=T(\Gamma)$,

When the distribution of random variable is nonnormal, the equivalent normal value of the mean and standard deviation for each non-normal random variable should be computed. For this aim, suppose that a particular random variable $X$ with mean $\mu_{X}$ and standard deviation $\sigma_{X}$ is described by a cumulative distribution function $(\mathrm{CDF}) F_{X}(x)$ and a probability 
www.IJRRS.com

density function (PDF) $f_{X}(x)$. To obtain the equivalent normal mean $\mu_{X}^{e}$ and standard deviation $\sigma_{X}^{e}$, we require that the CDF and PDF of the actual function be equal to the normal CDF and normal PDF at the value of the variable $x^{*}$ (design point) on the failure boundary described $g=0$. Mathematically, these requirements are expressed as $[13,14]$,

$F_{X}\left(x^{*}\right)=\Phi\left(\frac{x^{*}-\mu_{X}^{e}}{\sigma_{X}^{e}}\right)$

$f_{X}\left(x^{*}\right)=\frac{1}{\sigma_{X}^{e}} \phi\left(\frac{x^{*}-\mu_{X}^{e}}{\sigma_{X}^{e}}\right)$

Where $\Phi$ is the CDF for the standard normal distribution and $\phi$ is the PDF for the standard normal distribution. By manipulating these equation, we can obtain expressions for $\mu_{X}^{e}$ and $\sigma_{X}^{e}$ as follows:

$\mu_{X}^{e}=x^{*}-\sigma_{X}^{e}\left[\Phi^{-1}\left(F_{X}\left(x^{*}\right)\right)\right]$

$\sigma_{X}^{e}=\frac{1}{f_{X}\left(x^{*}\right)} \phi\left(\frac{x^{*}-\mu_{X}^{e}}{\sigma_{X}^{e}}\right)$

The main aim is to calculate $\beta_{H L}$ with Harmony Search evolutionary algorithm in space $\Lambda$. To achieve this, one has to solve a constrained optimization problem that is

$\left\{\begin{array}{l}\text { Minimize } \sum_{i=1}^{n} u_{i}^{2} \\ \text { Subject to } G\left(T^{-1}(u)\right)=0 .\end{array}\right.$

Solving Eq. (6) is equivalent to solving the relaxed form obtained by penalty method

$\left\{\underset{u}{\text { Minimize }} \sum_{i=1}^{n} u_{i}^{2}+\lambda \zeta\left(G\left(T^{-1}(u)\right)=0\right)\right.$,

Where $\zeta$ is the penalty function and $\lambda$ is the penalty coefficient (strictly positive). The solution $u^{*}$ of Eq. (6) or (7) is called the design point and enables us to calculate the reliability index as

$\beta_{H L}=\left\|u^{*}\right\|$.

The choice of the penalty coefficient $\lambda$ in Eq. (7) is crucial for the convergence of the search toward the solution of Eq. (6). In case of equality constrained as it is addressed in this paper, the penalty coefficient will be searched by an iterative process from a low value because the search space is a hyper-surface. According to [7], an appropriate sequence of $\lambda$ is $\lambda_{i}$, such that $\lambda_{i+1}=2 \lambda_{i}$ and $\lambda_{0}=0.1$. The value of $\lambda$ will be considered suitable when the quantity $\xi\left(G\left(T^{-1}(u)\right)\right)$ In Eq. (7) is small enough $\left(<10^{-4}\right.$ for example). For each problem solved in this paper, the corresponding $\lambda$ and the control parameter $\xi\left(G\left(T^{-1}(u)\right)\right)$ are supplied.

\section{Improved Harmony Search}

HS algorithm is based on natural musical performance processes that occur when a musician searches for a better state of harmony, such as during jazz improvisation. Jazz improvisation seeks to find musically pleasing harmony (a perfect state) as determined by an aesthetic standard, just as the optimization process seeks to find a global solution (a perfect state) as determined by an objective function [15].

The pitch of each musical instrument determines the aesthetic quality, just as the objective function value is determined by the set of values assigned to each decision variable. The HS algorithm works as follows:

Step 1. Initialize the problem and algorithm parameters.

The optimization problem is defined as Minimize $f(x)$ subjected to $X_{i L} \leq X \leq X_{i U}(i=1, \ldots, N) . X_{i L}$ and $X_{i U}$ are the lower and upper bounds for decision variables. The HS algorithm parameters are also specified in this step. They are the harmony memory size (HMS), or the number of solution vectors in the harmony memory; harmony memory considering rate (HMCR); bandwidth ( $b w)$; pitch adjusting rate (PAR); and the number of improvisations $(\mathrm{K})$, or stopping criterion.

Step 2. Initialize the harmony memory (HM). The initial harmony memory is generated from a normal distribution in the ranges $\left[X_{i L}, X_{i U}\right],(i=1,2, \ldots N)$ as shown in Eq. (9):

$H M=\left(\begin{array}{ccc}x_{1}^{1} & \cdots & x_{N}^{1} \\ x_{1}^{2} & \cdots & x_{N}^{2} \\ \vdots & \ddots & \vdots \\ x_{1}^{H M S-1} & \cdots & x_{N}^{H M S-1} \\ x_{1}^{H M S} & \cdots & x_{N}^{H M S}\end{array}\right)$

Step 3. Improvise a new harmony.

Generating a new harmony is called improvisation. The New Harmony vector $x^{\prime}=\left(x_{1}^{\prime}, x_{2}^{\prime}, \ldots, x_{3}^{\prime}\right)$ is determined by three rules: memory consideration, pitch adjustment and random selection. The procedure works as follows:

Foreachi $\in[1, N]$ do

Ifrand () $\leq H M C R$ then

$x_{i}^{\prime}=x_{i}^{j}(j=1,2, \ldots, H M S) \%$ memory consideration

ifrand $\leq P A R$ then

$x_{i}^{\prime}=x_{i}^{\prime} \pm r \times b w \%$ pitch adjustment

if $x_{i}^{\prime}>x_{i U}$

$x_{i}^{\prime}=x_{i U}$

elseif $x_{i}^{\prime}<x_{i L}$

$x_{i}^{\prime}=x_{i L}$

end 
www.IJRRS.com

end

else

$x_{i}^{\prime}=x_{i L}+\operatorname{rand}() \times\left(x_{i U}-x_{i L}\right) \%$ random selection

end

end

$x_{i}^{\prime}(i=1,2, \ldots, n)$ is the $i$ th component of $x^{\prime}$, and $x_{i}^{j}(j=$ $1,2, \ldots, H M S)$ is the $i$ th component of the $j$ th candidate solution vector in HM. Both $r$ and $\operatorname{rand}()$ are uniformly generated random numbers in the region of $[0,1]$, and $b w$ is an arbitrary distance bandwidth.

Step 4. Update harmony memory.

If the fitness of the improvised harmony vector $x^{\prime}=\left(x_{1}^{\prime}, x_{2}^{\prime}, \ldots, x_{3}^{\prime}\right)$ is better than that of the worst harmony, replace the worst harmony in the HM with $x^{\prime}$. Step 5. Check the stopping criterion.

If the stopping criterion (maximum number of iterations $K$ ) is satisfied, computation is terminated. Otherwise, Step 3 is repeated.

The most important step of the HS algorithm is Step 3 , and it includes memory consideration, pitch adjustment and random selection. $P A R$ and $b w$ have a profound effect on the performance of HS. Mahdavi et al. (2007) proposed a new variant of HS, called the improved harmony search (IHS) [15,21]. IHS dynamically updates $P A R$ and $b w$ according to Eqs. (10) and (11):

$$
\begin{aligned}
& P A R(k)=P A R_{\text {min }}+\frac{P A R_{\text {max }}-P A R_{\text {min }}}{N I} \times k \\
& b w(k)=b w_{\text {max }} \exp \left(\frac{\ln \left(\frac{b w_{\text {min }}}{b w_{\text {max }}}\right)}{N I} k\right)
\end{aligned}
$$

Where $N I$ is the maximum number of iteration, and $k$ is the current number of iterations; $P A R_{\min }$ and $P A R_{\max }$ are the minimum adjusting rate and the maximum adjusting rate, respectively; $b w_{\min }$ and $b w_{\max }$ are the minimum bandwidth and the maximum bandwidth, respectively. Parameters of the algorithm used in this paper for Corrosion reliability assessment are presented in Table 1.

Table 1. IHS parameters used for test problems

\begin{tabular}{|l|l|}
\hline Parameter & Value \\
\hline PAR & 0.3 \\
\hline HMCR & 0.9 \\
\hline PAR_min & 0.35 \\
\hline PAR_max & 0.99 \\
\hline bw_min & $1 \mathrm{e}-6$ \\
\hline bw_max & 1 \\
\hline NI & 990 \\
\hline HMS & 100 \\
\hline
\end{tabular}

\section{Pipeline Reliability Assessment}

Corrosion is a major problem for steel pipeline despite considerable effort and material expenditures spent in the last four decades on their failure prevention or in research [16,17]. Coating and cathodic protection failure do occur because of variety of causes leading to localized corrosion. As a consequence, there have been several techniques such as those specified in B31G [18] or Z184 [19] as well as hydrostatic test to estimate the remaining strength of a localized corrosion defect in a pipeline. Among the available techniques, B31G is the most widely used or accepted technique [20].

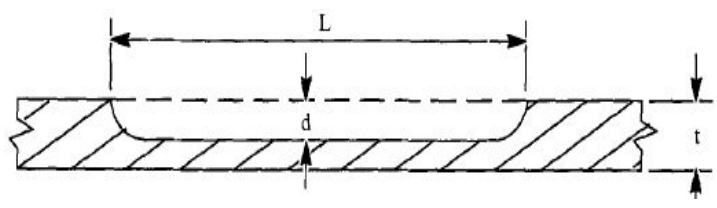

Fig. 2. Corrosion defect parameters

For comparison purposes, the B31G equation for predicting the burst pressure of corroded pipelines is also considered in the reliability and sensitivity analysis [3]:

$$
\begin{aligned}
& P_{b}^{B 31 G}=P_{b i}\left[\frac{1-(2 / 3)(d / t)}{1-(2 / 3)(d / t) M^{-1}}\right] \\
& M=\sqrt{1+0.8\left(\frac{L}{D}\right)^{2}\left(\frac{D}{t}\right)}
\end{aligned}
$$

where $\mathrm{L}$ is the axial length of the defect projected onto the pipe, $d$ is the average depth of corrosion, $t$ is pipe thickness in $\mathrm{mm}$ and $\mathrm{M}$ is Folias factor. The $\mathrm{d}$ parameter can be made more realistic by taking a sufficient number of depth measurements inside the corroded region. In B31G method, it is suggested that the area of metal loss (A) is calculated from the overall axial length and maximum depth of the corroded area and by considering the corroded area as either a rectangle or a parabola. Although the parabolic method was found preferable to the rectangular method, both methods were found to consistently fail to correctly estimate the actual failure stress.

To assess the probability of burst of a pipe with corrosion defects, it is necessary to relate the values of the operating internal pressure with the pipeline burst pressure. The corresponding limit-state function can be written as follows: where $P_{b}$ is the burst pressure of the corroded pipe and $P_{0}$ is the internal operating pressure [20]. Thus, the limit-state function is given by

$$
\begin{aligned}
& g(X)=P_{b}-P_{0} \\
& g(X)=\left(\frac{1.1 \sigma_{y} 2 t}{D}\right) \times \\
& {\left[1-0.9435(d / t)^{1.6}(l / D)^{0.4}\right]-P_{0}}
\end{aligned}
$$


www.IJRRS.com

In order to perform the corrosion reliability assessment of steel pipeline by the proposed algorithm and to validate its application, Statistical properties of random variables related to Khash Water transporting steel pipe are presented in Table 2. The result has also been compared with classical methods such as Monte Carlo Simulation method (MC).

As stated in the previous section, tuning of the penalty parameter is crucial, it is why its value used for the convergence is supplied in this paper for each addressed example. The value of $\xi\left(G\left(T^{-1}(u)\right)\right)$ Eq. (7) must be close to 0 . It will be considered close to 0 if its absolute value is lower than $10^{-4}$. This is a necessary condition to have the global optimum.

\section{Results and Discussion}

The history of Reliability index convergence for corroded pipe is shown in figures 1 and 2. Also, the probability failure of the considered steel pipeline obtained by proposed HSA and MC method is presented in Table 3.

It is clear that the proposed algorithm gives a good agreement with the approximation method of the more time-consuming method of Monte Carlo simulation, which is usually considered as the benchmark method.

Table 2. Statistical properties of variables

\begin{tabular}{|c|c|c|c|c|}
\hline Var. & Dist. & $\begin{array}{c}\text { Bias } \\
\text { factor }\end{array}$ & Mean & Std. dev. \\
\hline $\mathrm{d}$ & normal & 1 & $1.8 \mathrm{~mm}$ & $0.09 \mathrm{~mm}$ \\
\hline $\mathrm{l}$ & normal & 1 & $200 \mathrm{~mm}$ & $12 \mathrm{~mm}$ \\
\hline $\mathrm{t}$ & normal & 1 & $6.7 \mathrm{~mm}$ & $0.07 \mathrm{~mm}$ \\
\hline $\mathrm{D}$ & normal & 1 & $7000 \mathrm{~mm}$ & $0.51 \mathrm{~mm}$ \\
\hline$\sigma_{y}$ & $\begin{array}{c}\text { Log. } \\
\text { normal }\end{array}$ & 1.1 & $420 \mathrm{Mpa}$ & $39 \mathrm{Mpa}$ \\
\hline$P_{0}$ & Gumbel & 1 & $10 \mathrm{Mpa}$ & $0.1 \mathrm{Mpa}$ \\
\hline
\end{tabular}

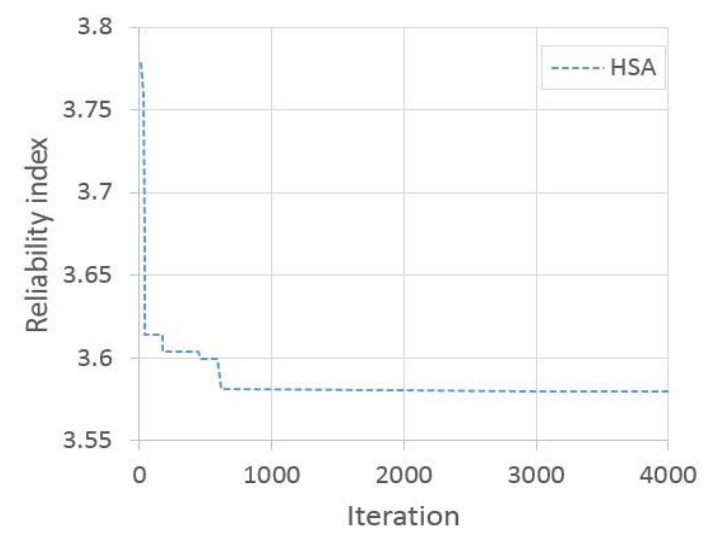

Fig. 3. History of beta convergence of proposed pethod (Iteration)
Figures 5 and 6 show the ratio between reliability index to depth of corrosion and internal pressure, respectively. It is clear that by increasing the internal pressure and depth of corrosion, probability failure of pipelines has increased too, but rates of reduction for reliability indices of these variables are different. So that by decreasing the reliability index from 5.25 to 4.5 , corrosion depth and internal pressure variation rates approach to 10.7 and 18.5 percentage, respectively. Also, maximum internal pressure and depth of corrosion can be obtained using these figures. For this purpose, we used two levels of failure (low failure probability or LFP with $\beta=5.25$ and medium failure of probability or MFP with $\beta=4.5$ ). Maximum internal pressures of this pipeline for the two mentioned levels of failure were obtained 11.67 and 13.98 . Furthermore, maximum depth of corrosions achieved for these levels of reliability are 0.72 and 1.24, respectively.

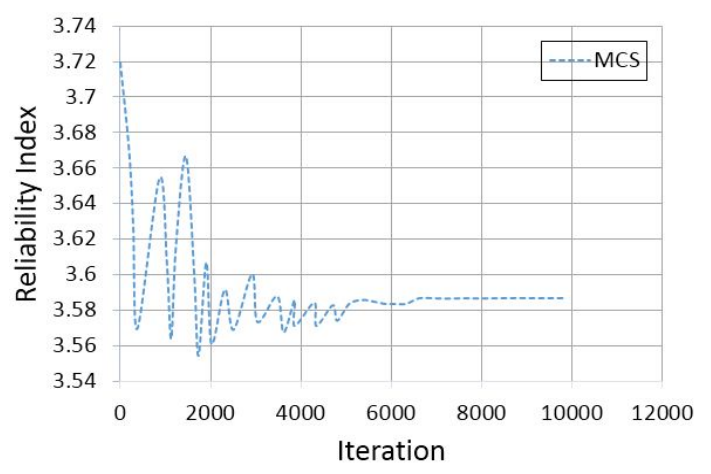

Fig. 4. History of Beta convergence for MCS (iteration)

Table 3. Obtained resultsof reliability index

\begin{tabular}{|c|c|c|c|}
\hline Method & $\begin{array}{c}\text { Reliability } \\
\text { Index }\end{array}$ & $\begin{array}{c}\text { Failure } \\
\text { Probability }\end{array}$ & Iteration \\
\hline Monte Carlo & 3.5869 & $0.161 \times 10-3$ & 6621 \\
\hline $\begin{array}{c}\text { Proposed } \\
\text { Algorithm }\end{array}$ & 3.5803 & $0.166 \times 10-3$ & 617 \\
\hline
\end{tabular}

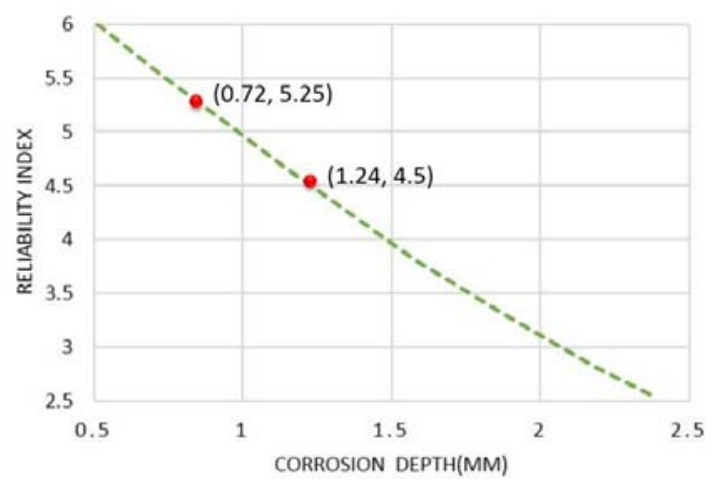

Fig. 5. Reliabilty index against d 


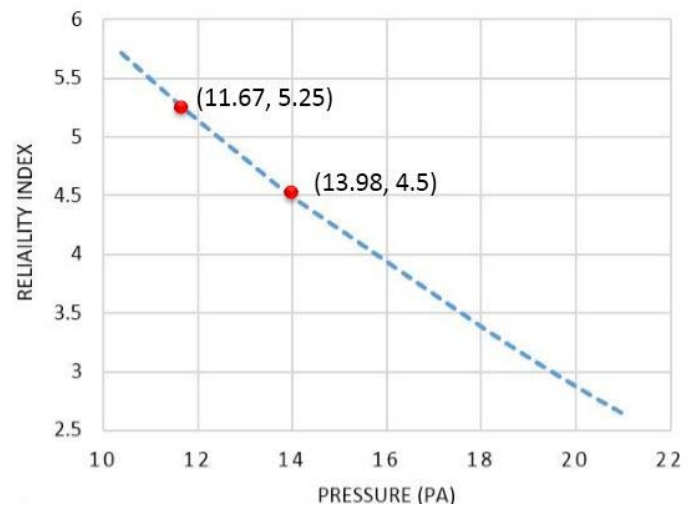

Fig. 6. Reliabilty index against internal P0

\subsection{Sensitivity Analysis}

In this section, sensitivity analysis of associated parameters is carried out to measure the effectiveness of each parameter on the probability of pipeline failure. Fig. 7 shows reliability index variation rate against coefficient of variation of random variables. Among the random variables, maximum difference of reliability index is achieved from depth of corrosion and pipeline thickness. Also, it is obvious that by increasing C.O.V of corrosion length, there is no significant change in reliability index. So, it can be considered as a deterministic variable with a standard deviation close to 0 . Next variable that plays the second level of importance and affects the reliability of pipeline is Thickness. Most variation of failure probability in return of this variable obtained from $\mathrm{COV}$ is less than 0.3. As shown in Fig.7, changes of diameter and internal pressure have a similar pattern, approximately. Variation of beta index against yield stress (Fy) starts when COV of Fy takes values less than 0.1 . As it can be seen, reliability index decreased dramatically from 3.5 at 0.1 to 1 at 0.6 that shows the importance of this parameter and the sensitivity of beta index to this variable.

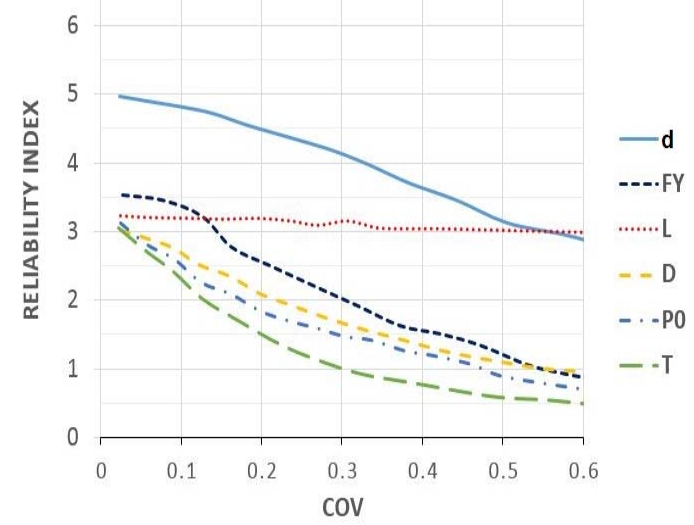

Fig. 7. Reliabilty index against variation of random variable COV
Fig. 8 shows maximum value of load applied to pipeline (internal pressure) against depth of corrosion to pipe thickness $(\mathrm{d} / \mathrm{t})$, according to the mentioned level of reliability.

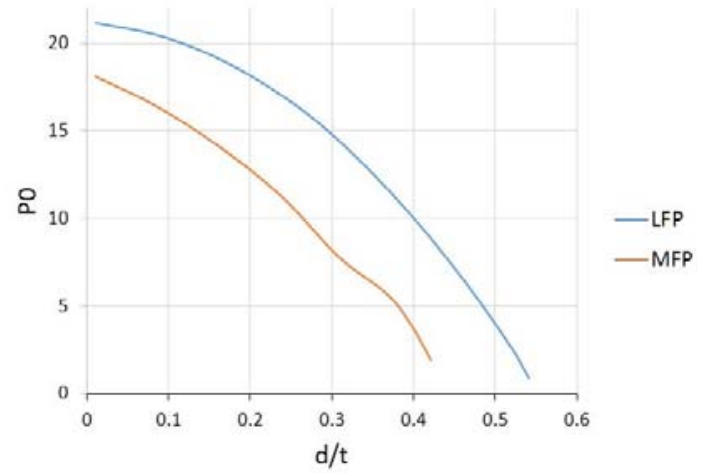

Fig. 8. Applied load against proportion of depth of corrosion to pipe thickness

Variation of tolerable pressure for the values of corrosion depth to thicknesses less than 0.1 is negligible (about $2 \mathrm{~Pa}$ ). Curve slopes of reliability level increased for the values of corrosion depth to thicknesses more than 0.2 that shows the intensity of failure for this range. In addition tocorrosion, reliability of pipes depends on their thickness. In fact, the pipelines can be better utilized for values of less than $0.1(\mathrm{~d} / \mathrm{t})$; otherwise, repairing, replacement or reduction of applied load (pressure) would be crucial.

\section{Conclusion}

Reliability methods can be considered as reliable ways for health monitoring and failure probability prediction of infrastructures to propose a confident scheme for utilization and maintenance of the existing and new pipelines. In this paper, a non-linear state model has been used for the structural analysis of corroded pipelines stressed by external forces. External load and substance corrosion have beenconsidered simultaneous base on limit state function and harmony search metaheuristic optimization algorithm has been selected for relaibility assessment instead of the time-consuming MC method, not only because of the advantage of its facility of implementation, but also for the possibility to deal with high non-liner and discontinuse limit state functions and capability of obtaining reliability index and failure propability without any need to derivaition. It can be seen that the result yielded is quasi-exact with respect to that yielded by MC classical methods.

Failure probability and reliability assessment have been obtained for the pipes used in Karvandar to Khash water transporting pipeline. Medium and low levels of failures defined and according to these levels, 11.67 and 
13.98 resulted as maximum of internal pressures. Corrosion length random variable does not have a significant effect on failure (for values greater than pipe radius) and can be considered as a deterministic variable. Whereas depth of corrosion has visible and remarkable affect, qua with increasing of $d$, failure severity increases. It can be concluded that for a less than 0.1ratio of corrosion depth to thickness, verified pipe is at an acceptable level of utilization. Otherwise, repair, replacement or reduction of applied load is proposed and for values greater than 0.3 , pipe replacement is strongly recommend.

\section{References}

[1].Kiefner, JF., "Failure stress levels of flaws in pressurized cylinders. ASTM STP 536, American Society for Testing and Materials, 1973, pp. 461-81.

[2].Kiefner, JF, Vieth, PH., "Evaluating pipe 1: new method corrects criterion for evaluating corroded pipe," Oil Gas $J$, Vol. 88, No. 32, 1990, pp. 56-9.

[3]. ASME B31G. Manual for determining the remaining strength of corroded pipelines. A supplement to ANSI/ASME B31G Code for Pressure Piping, press ASME, 1991.

[4]. DNV. Corroded pipelines recommended practice. Det Norske Veritas, RP-F101, 1999.

[5].Nahal, M. and Khelif R. Failure, "Probability Assessment for Pipelines under the Corrosion Effect," American Journal of Mechanical Engineering, Vol. 2, No. 1, 2014 pp. 15-20.

[6].Ditlevesen, O., Madsen, HO. "Structural reliability methods. Press Willey J, ISBN 0471960861, 1996.

[7]. Kazemi Eilaki N., Shabakhty, N. and Kia, M.A., "Seismic Reliability Assessment of Structures Using Harmony Search Algorithm," in National Conference on Earthquake, Structures and Computational approach, Kerman, Iran. 19-20 Oct. 2011, paper cescm_332, 175184.

[8]. Rubinstein, RY., Simulation and Monte Carlo Method, New York, Press Wiley, 1981.
[9]. Roy, D. and Dasgupta, T.A., "Discretizing Approach for Evaluating Reliability of Complex Systems Under Stress Strength Model,” IEEE Trans Reliable, Vol. 50, No. 2, 1981, pp. 145-50.

[10]. Zhao Y-G, "Ono AT.A general procedure for first/second order reliability methods (FORM/SORM)," Structural Safety J, Vol. 21, 1999, pp. 95-112.

[11].Rackwitz R. "Reliability analysis - a review," Structural Safety J, 2001, 23:365-3951.

[12].Nataf A. Détermination des distributions dont les margessontdonne'es, Compte Renduacade'mie des sciences, Paris, France, 1962, p. 225.

[13].Andrzei S. Nowak, Kevin Collins. Reliability of Structures. Press: McGraw-Hill Science/ Engineering/ Math; 1 edition, 2000.

[14].Ashgar B., "Nonlinear constrained optimization using penalty function,” Mathematical Developer Conference proceedings, 1999.

[15].Mahdavi, M., Fesanghary, M., and Damangir, E., “An improved harmony search algorithm for solving optimization problems," Applied Mathematics and Computation J, 2007, 188(2), pp. 1567-1579.

[16].Ramesh, S., Pipeline Reliability Assessment. Publisher: Press Gulf Professional, ISBN-978-0-12-813045-2, 2017.

[17].Amirat A., Benmoussat A. and K. Chaoui. Reliability Assessment of Underground Pipelines Under Active Corrosion Defects. Damage and Fracture Mechanics: Failure Analysis of Engineering Materials and Structures, 2009, pp.83-92.

[18].Manual for Determining the Remaining Strength of Corroded Pipelines. Press ASME, 2017.

[19].CAN/CSA-Z184-M92. Gas Pipeline Systems, Press Standards Council of Canada, ICS Codes: 23.040.01, 1992.

[20].Teixeira, A.P., Soaresa, C.G., Nettob, T.A., "Estefenb S.F.Reliability of pipelines with corrosion defects," Journal of IJPVP, Vol. 85, 2008, pp. 228-237.

[21] Kazemi Eilaki N., Shabakhty N. and Kia M.A. and Sanayee Moghadam S., "Structural Reliability: An Assessment Using a New and Efficient Two-Phase Method Based on Artificial Neural Network and a Harmony Search Algorithm," Civil Engineering Infrastructures Journal, Vol. 49, No. 1, 2016, pp. 1-20. 